\title{
Sexism and Sexual Harassment in Medicine: Unraveling the Web
}

\author{
Anitha Menon, BSE®
}

University of Michigan Medical School, Ann Arbor, MI, USA.

J Gen Intern Med 35(4):1302-3

DOI: $10.1007 / \mathrm{s} 11606-019-05589-0$

๑) Society of General Internal Medicine 2019

$\mathrm{T}$ he 2018 report from the National Academy of Sciences, Engineering, and Medicine, which found that female medical students are much more likely to experience sexual harassment than our peers in other STEM disciplines, has only revealed and validated what women in medicine have endured since they entered the workforce. ${ }^{1}$ The report emphasizes the link between sexual harassment and poor professional outcomes, like job dissatisfaction and reduced productivity, and psychological outcomes, like depression and lowered life satisfaction, and it has pushed the medical community to identify solutions to sexual harassment such as changes to institutional policy and trainings for faculty and staff. ${ }^{1}$ But the discourse on this significant problem has largely focused on "eliminating" sexual harassment in a vacuum, chopping it from its roots in sexism. In the 2 years I've spent studying this topic, I've sometimes wondered if my colleagues recognize that sexual harassment is a manifestation of institutional sexism that is embedded in our culture, not just a cancer that emerged suddenly, de novo. Perceptions of what is and what is not sexual harassment vary immensely between students, residents, and attendees, based on the dominant culture of one's training environment and personal identity. And, ironically, the very power dynamics that enable sexual harassment to persist also prevent open dialogue due to fear of retribution. So while brainstorming responses to sexual harassment is tempting, if we do not fully explore its manifestations and characterize its causes, we risk implementing solutions that manage symptoms without treating the disease.

To understand women's experiences of sexual harassment in medicine, one must first recognize that from childhood, women are primed by sexist norms and stereotypes that define us as sexual objects and caregivers. ${ }^{2}$ Prior to the passage of Title IX in 1972, few women became physicians; our expected roles were predominantly in the home. ${ }^{3}$ And although sexism stems from

Prior Presentations None

Received June 26, 2019

Accepted November 27, 2019

Published online December 12, 2019 society, academic medicine is rife with distinctive power structures that institutionalize the problem. Some of these structures are shared with other professions like biased hiring and advancement processes that block women from leadership positions, tenure clocks that conflict with women's biological clocks, and unsupportive maternity, breastfeeding, and childcare policies; others are more unique, like hierarchical and male-dominated training environments and a lack of woman mentors and sponsors due their under-representation as senior medical faculty. 1,4 These power structures intersect to create suffocating webs that ensnare all those who exist within the system except those at the center. Sexual harassment is a manifestation of these invisible power structures. Overt sexual advances are just the tip of the iceberg, with gender harassment, a range of behaviors "not aimed at sexual cooperation but that convey insulting, hostile, and degrading attitudes about women," being the most common and insidious form of sexual harassment. ${ }^{5}$

I became acutely aware of the tangled webs that restrict me during my first clinical rotation. I was appalled when a middleaged male patient looked me up and down and barraged me with questions (how old are you? Are you married? Where are you really from?). Despite my discomfort, I did not want to appear weak or emotional by leaving the room so I stoically continued the interview. Once I began to ask about his chief complaint ("groin bulge"), he immediately unbuckled his belt and offered to take his pants off and show me his testicular bulge. This was one of the first times I had interviewed a patient alone, and I had no idea how to respond. Trying to remain professional, I asked him to please wait until I got the attending and left. When we returned to the exam room, the attending gestured for me to begin the testicular exam. I had barely put gloves on before the patient looked at the attending and asked, "So where can I get myself one of these pretty assistants?" To my dismay, the attending — without missing a beat-replied, "Well, they send them to us every month - they're medical students." I opened my mouth only to find I was at a loss for words; in that moment, I was both too visible and invisible. Eyes brimming with tears, I completed the exam. To this day, I wish I had not.

My clinical rotations were polluted with incidents that repeatedly reminded me of my status as a woman in medicine: a patient asking me for my phone number over and over again in front of my resident; another attending telling the operating room nurse while draping a patient before surgery he wanted the patient's nipples exposed because he "had a fetish"; another attending telling me this was a culture I had to "grow a thick 
skin to." I began to feel disillusioned, burned out, and wary of male patients and, to an extent, male physicians. The common thread between these incidents is there were almost always other people in the room and all were either peers or seniors. Why did they remain silent? What webs were they also entangled in?

When I tried to become involved in solutions, I realized the complexity of the problem. I was struck by a point made by a research mentor who studies these issues extensively: sexual harassment, especially in the form of gender harassment, is rarely about sex - it is about power. ${ }^{6}$ It reinforces a gender hierarchy that has become normative and serves to remind women that we are seen as objects, not physicians.

When one describes sexual harassment as an action that is cleansed of its dependent relationship to sexism, it is at times difficult to call inappropriate behavior "sexual harassment" and at other times too easy, particularly when a patient is the perpetrator. Given that cultural definitions of sexual harassment have changed significantly since many senior faculty were trainees, there is often not a consensus on what constitutes sexual harassment in clinical settings or why it occurs. At a training on sexual harassment, an attending told me that a man calling his female doctors "distractingly beautiful" is a compliment and that women should learn how to take compliments because this is part of connecting with patients. Another attending said that this is no different from a male patient complimenting his haircut. Then, one man predictably told the story of a woman calling him handsome during rounds as his personal story of sexual harassment.

But most women who have been called attractive by a patient know that this is not a compliment-rather, it is a reminder that we are valued in society for our looks rather than our competence. A man commenting on his male doctor's haircut is devoid of that invalidation because for the entire history of medicine men have always been the physicians and have always been valued for their intelligence. And while women can be inappropriate to men-and this behavior certainly should not be condoned - this is not an extension of systematic devaluation, as it is for women. For women of color (particularly Black, Latina, and Native women) and transgender individuals, this devaluation is manifold worse given their under-representation in medicine due to institutional racism and gender norms. Accepting these false equivalencies gives room for debates on "reverse sexism," a term that ignores the webs of power that create and underpin "sexism."

I am passionate about the medical community addressing sexual harassment. But to do this, we should partner with or follow the lead of advocacy organizations like Time's $U p$ Healthcare to educate our communities on what sexual harassment is and why it is related to inequity. ${ }^{7}$ Sexual harassment cannot be explained by "bad actors," but rather by issues in the broader culture. ${ }^{6}$ When we talk about sexual harassment, we must discuss gender inequity, the limited professional support for women physicians who are also parents, the lack of leadership opportunities for women (women hold only $16 \%$ of permanent deanships at accredited medical schools), and a lack of institutional support and transparency about sexual harassment reporting. ${ }^{1,4}$ We must be brave by engaging our colleagues and superiors in these difficult conversations and challenging their false equivalencies in a productive way. And finally, we must listen to women instead of silencing them with rebukes like, "Just don't let it get to you" or "Just take the compliment."

In 1 year, the other women in my class and I will graduate from medical school. Having an MD and being leaders of our teams and organizations will give us the power to advance gender equity for woman physicians and, by extension, other health professionals. But we need allies. Allies may not be impacted by sexual harassment in the same way (men, white people, cis people), but they are willing to center women's voices and educate themselves and their communities on how sexism and other inequities are not behaviors but structures of power that individuals reinforce through the things they do and the things they fail to do. In order to resist institutional sexism and the other webs that keep us in our place, we as a field must acknowledge their existence by naming them. Only then can we begin the slow work of unraveling them.

\section{Acknowledgments:}

Contributors: Reshma Jagsi, $M D, D P h i i^{1,2,3}$ : for outstanding mentorship and reviewing the manuscript

${ }^{1}$ University of Michigan Medical School

${ }^{2}$ Center for Bioethics and Social Sciences in Medicine, University of Michigan

${ }^{3}$ Department of Radiation Oncology, University of Michigan

Dr. Jagsi contributed significantly to this work and provided written consent to be included in the "Acknowledgments" section of this manuscript.

Corresponding Author: Anitha Menon, BSE; University of Michigan Medical School, Ann Arbor, MI, USA (e-mail: avmenon@med.umich. edu).

\section{Compliance with Ethical Standards:}

Conflict of Interest: The authors declare that they do not have a conflict of interest.

\section{REFERENCES}

1. National Academies of Sciences, Engineering, and Medicine. Sexual harassment of women: climate, culture, and consequences in academic sciences, engineering, and medicine: National Academies Press; 2018.

2. Leaper C. Gender and social-cognitive development. Handbook of child psychology and Dev Sci 2015:1-48.

3. Carnes M. What would patsy mink think? JAMA. 2012;307(6):571-2

4. Harp D, Shim RS, Johnson J, Harp JA, Wilcox WC, Wilcox JK. Race and gender inequalities in medicine and biomedical research. Critical Research on Sexism and Racism in STEM Fields: IGI Global; 2016. p. 115-34.

5. Fitzgerald LF, Gelfand MJ, Drasgow F. Measuring sexual harassment: theoretical and psychometric advances. Basic Appl Soc Psychol 1995; 17(4):425-45.

6. Cortina LM, Jagsi R. What can medicine learn from social science studies of sexual harassment? Ann Intern Med. 2018;169(10):716-7.

7. Choo EK, van Dis J, Kass D. Time's up for medicine? Only time will tell. New England J Med. 2018;379(17):1592-3.

Publisher's Note Springer Nature remains neutral with regard to jurisdictional claims in published maps and institutional affiliations. 